\title{
Consecutive Enzymes in the Glycolytic Pathway
}

EARLY crystallographic studies of globular proteins were carried out on proteins that could be obtained readily in large quantities, such as myoglobin, haemoglobin and extracellular enzymes like lysozyme and ribonuclease, and on them protein crystallographers developed an armoury of weapons with which they can now attack problems of increasing biochemical complexity. From being chiefly preoccupied with the tactics of structure determination, they can now afford to turn at least part of their attention to the strategy involved in learning as much as possible of the control processes of living systems. In place of the study of single enzymes in isolation, concerted attacks are now beginning in several laboratories on systems of enzymes such as those of the glycolytic pathway.

Enzymatic pathways are likely to have become more complex during evolution, additional enzymes having been introduced to give finer control, the specificity of each enzyme becoming narrower and the chemical change at each step becoming smaller; a structural similarity between neighbouring enzymes in a pathway might show that such evolution had taken place by gene duplication and divergence from a common ancestor. Efficient operation of the pathway might be promoted by direct interaction between enzymes, in which case it might be possible to see evidence of structural complementarity. More certainly, generally similar geometries might be expected in the substrate binding sites of neighbouring molecules, because the product of one enzyme is the substrate for the next. The subtle differences between binding sites, however, might be particularly informative in assessing the importance of distortion of the substrate conformation towards that of the transition state as a factor in rate enhancement by enzymatic catalysis.

The publication by Watson and his colleagues in this week's Nature New Biology (240, 130, 134 and 137 ; 1972) of preliminary studies of three consecutive enzymes from the glycolytic pathway represents another landmark in the development of protein structure analysis. While these papers contain some information of real importance, the crystallographer looks upon the successful calculation of a low-resolution map as having far more significance than the information that it directly reveals; it is an indication that the often frustrating search for pure protein, for suitable crystals for X-ray study and for the appropriate isomorphous heavy-atom derivatives have all been successful and that only a matter of time (and some effort) is required for a detailed picture to emerge.

The early stages of Watson's work on one of these enzymes, glyceraldehyde - 3 - phosphate dehydrogenase (GPDH), were by no means straightforward; the present work on the human enzyme was preceded by a lengthy and generally fruitless study of material from other species. Indeed even the human form has presented its special difficulties in the shape of a tendency for the crystals to twin, probably a consequence of the near but not exact 222 symmetry of the tetrameric molecule. Unfortunately, if this is the correct explanation, twinning may prove to be a recurrent problem in the study of oligomeric enzymes.

Watson, Duée and Mercer (Nature New Biology, 240, $130 ; 1972$ ) have been able to establish the overall, nearly spherical, shape of the GPDH molecule and to identify, at least tentatively, the positions of the boundaries between subunits. While crystal symmetry insists that the molecule has identical halves, the electron-density map shows that the two subunits in each half are also generally very similar. The similarity breaks down, however, in a localized region that lies close to the binding site of a heavy-atom compound known to inhibit the enzyme in solution. Watson et al. consider that it is premature to deduce too much from this observation, but it looks probable that there is an asymmetry of the subunits in their active site regions.

Comparison of the GPDH map with corresponding maps of lactate and malate dehydrogenases shows some possible similarities but also major structural differences. The true situation, together with the unequivocal location of the active site, is unlikely to be resolved until a more detailed map is available.

The structure analysis of yeast PGK, by Wendell, Bryant and Watson (Nature New Biology, 240, 134 ; 1972) has confirmed but added comparatively little to the picture of the PGK molecule from horse described earlier this year by Blake, Evans and Scopes (Nature, 235, 195 ; 1972). The molecule is a monomer of weight about 45,000 and in shape consists of two lobes connected by a narrow waist. Blake et al. found that $\mathrm{Mg}-\mathrm{ADP}$ bound to one lobe, but the other seems to be too remote to form part of the same active site; they suggested that the second lobe might be important in control or in binding to another molecule, perhaps even another glycolytic enzyme. Wendell et al. suggest now that the PGK molecule might bend at its waist, allowing both lobes to form the active site in solution, and they hint at a slight difference in the relative disposition of the two lobes in the yeast and horse PGK crystals. Undoubtedly the eventual comparison of the two enzymes at higher resolution will throw some light on this question as well as showing the sort of variation undergone by the enzyme through a long span of evolutionary history.

The structure of the third enzyme of the series, yeast phosphoglycerate mutase, reported by Campbell, Hodgson and Watson (Nature New Biology, 240, 137 ; 1972), is again tetrameric with one exact two-fold axis relating identical halves, the two subunits in each half appearing to be very similar in conformation. The contacts between the two subunits in each half are more numerous than those between the two halves, suggesting that the molecule might dissociate into dimers fairly readily, and it is of interest that the corresponding enzyme from muscle is only dimeric. Preliminary attempts have been made to locate the active site of the yeast enzyme, which has been little studied chemically; in particular, no aminoacid sequence information is yet available, and Watson and his colleagues hope that their progress on the structure may stimulate activity in these directions.

These structural results have not so far revealed any grand design of the enzymes in the glycolytic pathway, the only family likeness discernible at this stage being a rather doubtful one between GPDH and other dehydrogenases. Nevertheless, the three structures have some features of real interest and one can look forward with some assurance to detailed models of a number of glycolytic enzymes becoming available within the next year or two.-A. C. T. N. 